\title{
Industrial benchmarking and evaluation of ADDSAFE FDD designs
}

\author{
Philippe Goupil*, Andres Marcos** \\ *AIRBUS, Flight Control System, 316, route de Bayonne, 31060 Toulouse Cedex 09 \\ France (Tel: +33-561183803; e-mail: philippe.goupil@airbus.com). \\ **DEIMOS-SPACE S.L.U. Ronda de Poniente 19 Edificio Fiteni VI, P2, 2 Tres Cantos, Madrid 28760 \\ Spain (Tel: +34-918063462; e-mail: andres.marcos@deimos-space.com)
}

\begin{abstract}
In this article the industrial benchmarking and validation process used within a European Framework $7^{\text {th }}$ project termed "Advanced Fault Diagnosis for Sustainable Flight Guidance and Control (ADDSAFE)" is presented. This process is used with two main goals: (i) benchmark the Fault Detection and Diagnosis (FDD) designs developed within the first phase of the project, (ii) demonstrate the applicability of the proposed FDD techniques in a standardized industrial validation process in order to successfully transfer these techniques to the aeronautics practitioners. The article details the software tools, test-bench facilities, validation process and a summary of the application of the process to the developed ADDSAFE FDD designs.
\end{abstract}

Keywords: Aerospace Engineering, Flight Control, Fault Diagnosis, Validation, European Project.

\section{INTRODUCTION}

A consortium of European industrial partners, research establishments and Universities has been established with funding from the European Union 7th Framework Program (EU-FP7) to address the challenge of the future "sustainable" aircraft - i.e. Cleaner, Quieter, Smarter and More Affordable [1]. The overall aim of the project, termed "Advanced Fault Diagnosis for Sustainable Flight Guidance and Control (ADDSAFE)", is to contribute to the aircraft structural design and performance optimization thanks to advanced Fault Detection and Diagnosis (FDD) in the Flight Control System (FCS). More precisely, it can be demonstrated that improving the fault diagnosis performance in FCS allows to optimize the aircraft structural design (resulting in weight saving), which in turn helps improve aircraft performance and to decrease its environmental footprint (e.g. fuel consumption and noise).

The FDD challenge tackled in ADDSAFE consists mainly in sensor and actuator malfunctions [1]. Specifically, flight parameter management system, abnormal aircraft behaviour and servo-loop actuator and sensor fault: see references [617] for more details. The importance of the studies carried out within the project arises, on the one hand, due to the industrial representativeness of the benchmark proposed by Airbus [2], which consists of a generic civil aircraft model and realistic fault scenarios, and on the other hand, the industrial validation of the more promising designs in the actual Airbus flight control system Verification \& Validation $(\mathrm{V} \& \mathrm{~V})$ process.

This paper is dedicated to the industrial assessment of the FDD designs developed by the consortium to tackle the ADDSAFE industrial challenge. The validation is formed by a mix of industrial software assessment tools and physical aircraft rigs (e.g. Airbus iron-bird and flight simulator).

The layout of the paper is as follows. After a reminder on the typical industrial V-cycle, section 2 describes the validation tools and bench-tests employed in the ADDSAFE project: a software-based Functional Engineering Simulator (FES) and hardware-based industrial test facilities. Section 3 is dedicated to the description of the industrial criteria used for benchmarking the developed designs, which is used to select the most promising designs for further industrial validation. Section 4 details the industrial benchmarking and validation process. Section 5 summarizes the results from its application to the developed FDD designs. Section 6 ends with some concluding remarks and directions for future research.

\section{INDUSTRIAL VALIDATION MEANS}

The aircraft industrial development process is depicted in the V-cycle of Figure 1.

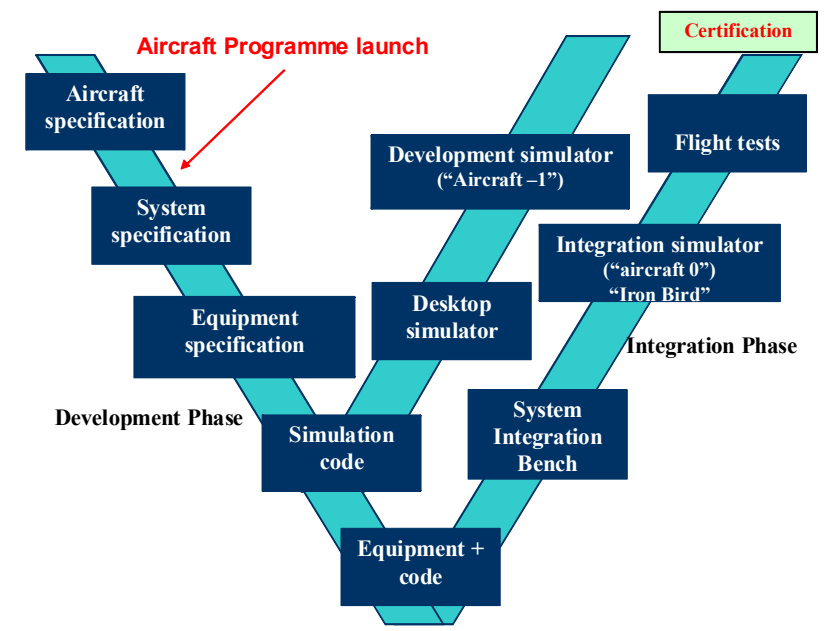

Figure 1: Aircraft development process, the V-cycle. 
The first branch of the V-cycle is the development phase. It starts with the aircraft specification corresponding to the "top level requirements": the definition of the needs, the choice of concepts, control laws, technologies, etc. The aircraft is decomposed into sub-parts, called systems, which are specified in the next step. The systems are decomposed in subparts called "equipment" (e.g. a Flight Control Computer, FCC), which are then specified. At this step, this specification can be used in a desktop simulator to fly the aircraft in its environment to check that it satisfies the performance and safety requirements before the associated code is even implemented in the equipment. This specification is also used in a development-simulator, a real cockpit where all systems and environment are simulated. After equipment specification, the corresponding flight code is generated and implemented in the hardware equipment. The second part of the V-cycle can then start. This integration phase consists of a severe validation campaign on different test benches, from the simplest ones (an actuator bench) to more complete ones (the "Iron Bird"). The validation phase ends with the flight tests and the overall V-cycle ends with the certification process.

ADDSAFE addresses the development and the integration phases, from FDD design coding to high-fidelity simulators (flight tests are not part of this project). Indeed, a key step for the successful transfer to the aeronautics practitioners of the developed FDD methods is their demonstration on standardized industrial validation processes. As already mentioned, the proposed validation is a two-steps process: first, an industrial software assessment tool (FES) is used and secondly, validation on physical aircraft rigs is performed.

This two-steps process allows performing a stringent validation campaign exploring the whole flight domain, a wide class of pilot inputs and taking into account perturbations and uncertainties (e.g. wind \& aerodynamics). It also allows using the state-of-the-art industrial validation on real test facilities for the selected FDD designs.

\subsection{Functional Engineering Simulator}

The Functional Engineering Simulator (FES), developed by Deimos Space S.L.U., is a non-real-time simulator based on Simulink, Matlab and XML that includes Airbus aircraft benchmark as well as robustness and performances analysis tools for all the fault scenarios defined in the project [1]. The FES is not currently part of the industrial V-cycle and is not depicted in Figure 1. However, it would be located towards the end of the development phase, between the simulation code generation and the implementation of the code in the equipment.

The FES is a term used in Space to describe a software simulator describing at a functional level the components of a system (including its operating environment). FES are used in support of the specification, design, verification and operations of space systems, and can be used across the spacecraft development life-cycle, including activities such as system design validation, software $V \& V$, spacecraft unit and sub-system test activities $[3,4]$.

The ADDSAFE-FES main objectives are: to provide a faithful simulation environment for the selected fault scenarios, and to support the development and benchmarking of the FDD designs. In particular, the FES allows performing intensive Monte-Carlo campaigns for assessing the robustness and performances of the designs proposed by the consortium.

Deimos has developed two FES packages. One serves as a simulation and verification FES released to all partners for their use during the development and application of the FDD methods, and the other for the industrial benchmarking and validation performed by the industrial partners, which includes more sophisticated tools for multi-team FDD designs' benchmarking.

In terms of output visualization and analysis, raw data plots can be used to show the output of all the Monte Carlo simulations besides the nominal simulation (in red), as illustrated in Figure 2. Once a simulation has been run, the raw simulation outputs can be post-processed to obtain new variables for the analysis of the system. Figures-of-Merit (FOM) are produced as scalar quantities that characterize the performance of the FDD system (cf. section 3).

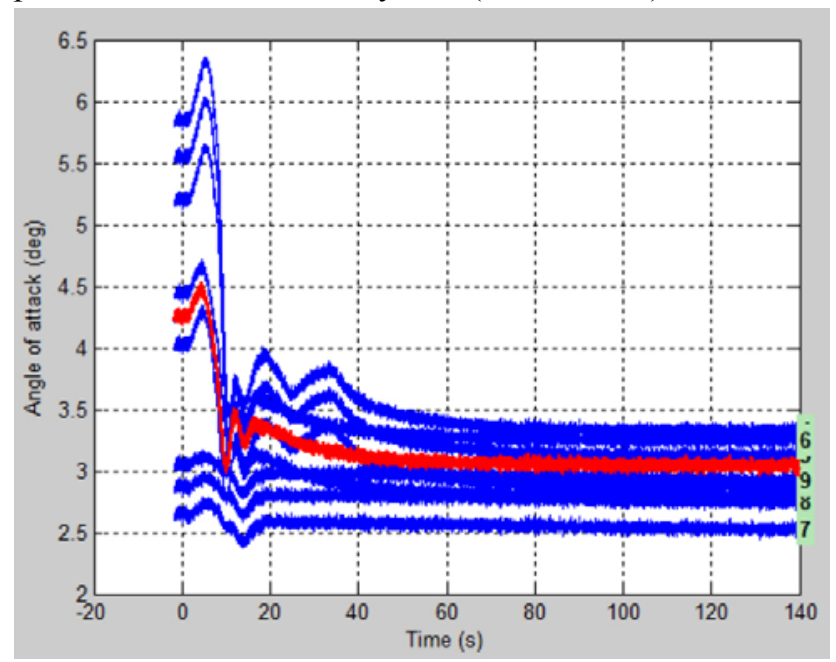

Figure 2: FES, parametric simulation plot

\subsection{Industrial Validation Test-Benches}

From an aircraft manufacturer point of view, all new types of equipment installed in the cockpit and in the aircraft avionics compartment must be tested, including checking their connection to the other aircraft equipments as well as their integration.

After a first assessment of the equipment itself (e.g. on a desktop simulator for validating a flight guidance and control function, Figure3.a), there are two levels of integration test facilities:

- $\quad$ The System Integration Test Bench (SIB, Figure 3.b) for validation in an environment restricted to a single, specific aircraft system function (e.g. FCS)

- The integration simulators ("Iron Bird" or flight simulator) for validation in the full aircraft environment.

In the Flight Control System environment, the SIB is a test bench with simulated inputs and observation of FCC internal variables. This bench offers the possibility of validating degraded configurations: e.g. low hydraulic pressure or high 
aerodynamic loads on the control surface. The so-called "Iron Bird" (Figure 3.c) is a kind of very light aircraft, without the fuselage, the structure, the seats, etc, but with all system equipment installed and powered as on an aircraft (e.g. hydraulic and electric circuits). And finally, the flight simulator (Figure 3.d) is a test bench with a real aircraft cockpit, flight control computers and coupled to a rigid aircraft model. The Iron Bird can also be coupled to the flight simulator. The levels of fidelity of each test-bench are explained in more detail in reference [18].

For the ADDSAFE project, the choice of the validation test facility depends on the characteristics of the FDD designs, and its associated fault scenario coverage, selected for industrial validation.

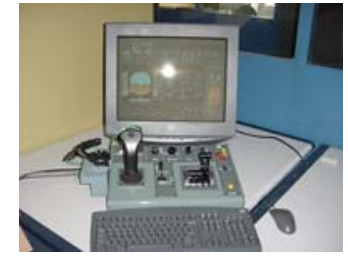

a) Desktop simulator

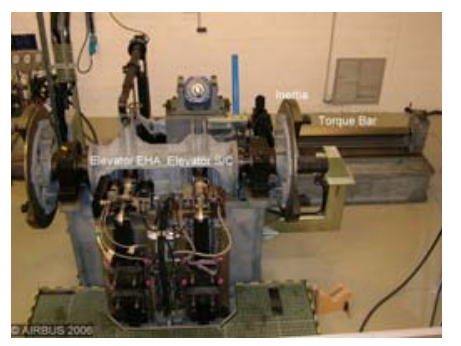

b) A380 elevator SIB

Figure 3: Airbus test facilities.

\section{INDUSTRIAL ASSESSMENT CRITERIA}

The purpose of this section is to list the quantitative and qualitative criteria used within the ADDSAFE project to evaluate and compare the FDD designs during the industrial validation phase. The quantitative component is given by metrics and a cost function which are automatically calculated by the FES for each FDD design based on a Monte Carlo campaign. The qualitative evaluation (based on Airbus FDD design and V\&V teams' experience) is used to assess the designs' practical implementation and relevance for industrial use.

\subsection{Quantitative assessment}

In order to define an evaluation and benchmarking matrix, a set of definitions are first presented based on Figure 4. These definitions facilitate the mathematical formulation of the selected evaluation metrics, and together with the defined cost function (Eq. 4) allow providing a hierarchy for the classification of the FDD designs based on their performance and robustness.

In Figure 4, the corresponding definition for each 'time' parameter is as follows:

- t_fault: time instance at which a fault is activated.
- t_detect: time instance at which a fault is declared as detected by the FDD system.

- t0: time instance at which a fault must have been declared by the FDD system, according to Airbus requirements.

- $\mathrm{T}_{\mathrm{D}}$ : detection time, time period between activation of a fault and its detection.

- $\mathrm{T}_{0}$ : maximum allowed detection time.

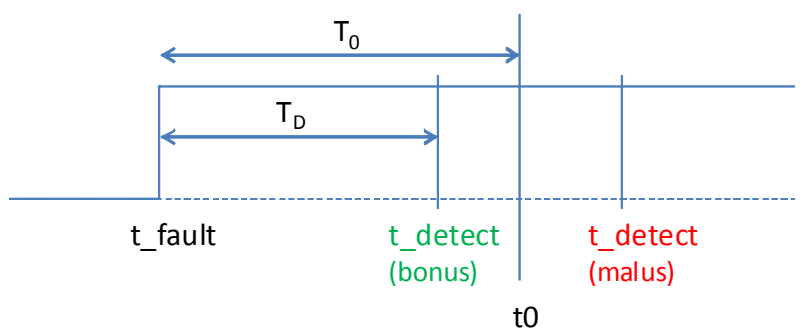

(the fault must be detected at t0)

Figure 4: Definition of fault and detection time parameters

With these definitions, the evaluation metrics can be defined. The Detection Time Performance (DTP) can be defined as:

$$
D T P=\frac{T_{D}}{T_{0}}=\frac{t_{-} \text {detect }-t_{\_} \text {fault }}{t_{0}-t_{-} \text {fault }}
$$

Statistics of this metric, such as average, minimum, maximum and variance values are calculated but the most important, from the industrial point of view and for assessment purposes, is its maximum value which corresponds to the worst case.

The False Alarm (FA) rate metric is computed taking into account the total number of cases yielding a false alarm $n_{F A}$ out of the total number of Monte-Carlo runs $n_{M C}$ :

$$
F A_{\%}=\frac{n_{F A}}{n_{M C}} .100
$$

In the same manner, the Missed Detection (MD) rate index is computed as the percentage ratio of MD cases $n_{M D}$ w.r.t. $n_{M C}$ :

$$
M D_{\%}=\frac{n_{M D}}{n_{M C}} .100
$$

An Executive Time (ET) metric is defined to represent the computational burden of the proposed designs. This metric is more oriented towards the FDD design viability (e.g. applicability) in a very stringent real-time environment. It is expressed in $\mu s$, representing the running time once a FDD design is coded in a real-time environment (see section 4 for more details). This metric is very useful for estimating the required percentage of the FCC CPU that is demanded for an implementation.

These four metrics are the main criteria used for assessing the robustness and performances of the proposed designs. All metrics are accurately numerically defined (not disclosed in this paper for confidentiality reasons): DTP is defined for each fault scenario, while FA and MD are defined taking into account the operational reliability requirements, and ET is characterized in relation to the FCC capacities.

A normalized cost function is computed, based on a metrics' weight performance (WP) and weight criticality (WC). The WP and WC reflect respectively satisfaction level per metric (dependent on the FDD system characteristic) and the metric criticality (independent of the FDD system, fixed by the 
industrial requirements). If a FDD design is strictly compliant with Airbus requirements, then it scores a WP equal to 3 . Thus, the following cost function is proposed, normalized with respect to a level of ' 3 ':

$$
\text { Cost }=\frac{\sum_{i=1}^{n} W P_{M i} W C_{M i}}{3 \sum_{i=1}^{n} W C_{M i}}
$$

If a FDD design is able to perform better than the industrial requirements then the cost will be greater than 1 . On the other hand, if a FDD design is unable to achieve the required metrics then the corresponding cost will be lower than 1 .

Note that only the maximum DTP is retained as a significant statistic for the cost function computation. Indeed, in view of a possible use in practical aerospace application the worst case must be taken always into account rather than an average behavior.

\subsection{Qualitative assessment}

A pure quantitative assessment is not sufficient for evaluating the industrial relevance of the proposed FDD designs. Base on its experience [18], Airbus has also defined some metrics conveying the potential of implementing a design on a real aircraft. These metrics are not part of the cost function due to the impossibility of numerically defining most of this implementation experience.

In particular, for bridging the gap between the basic research level arising from academic publications and the industrial needs, the proposed techniques must be clearly understood by the industrial engineering practitioners. Thus, the innovative FDD designs must be easily transferable to the industrial world, which means that the techniques must be easy to master and straight forward to tune, especially as a design may be used on different control surfaces and for different aircraft. A completely new and complex tuning required for each application is not acceptable from an industrial point of view. This is why, among other criteria, a set of tuning complexity metrics has been defined. This encompasses the following metrics [19]:

- The number of input parameters of the FDD design, with or without a physical meaning. This metric is of primary interest as it impacts the $\mathrm{V} \& \mathrm{~V}$ workload and consequently the system development duration.

- The physical meaning of these inputs parameters: in view of adapting the proposed design to another control surface and/or on a different aircraft, it is more interesting to use input parameters with a physical meaning.

- It is also industrially relevant to know if a clear procedure describing step by step how to tune the FDD design can be specified, describing for example how many steps are required for a high-level tuning.

Among the number of input parameters, the number of parameters to tune is also an interesting metric, taking into account that it is better to have ten parameters easy to tune rather than two with a large tuning complexity. The V\&V activities workload is also impacted as the effect of a bad tuning must be monitored.

\section{INDUSTRIAL VALIDATION CAMPAIGN}

\subsection{Monte-Carlo campaign}

As already mentioned, the first step of the industrial validation consists of a Monte-Carlo campaign performed using the ADDSAFE-FES, which results in the automatic computing of the metrics described in the previous section.

The evaluation process consists of applying a Monte Carlo campaign with a high number of $n_{M C}$ runs decomposed into two cases:

- $n_{M C F F}$ fault-free runs distributed evenly among all the proposed benchmark flight manoeuvres (see [1] for more details). These runs are dedicated to the assessment of the FDD design robustness.

- $n_{M C W F}$ runs at the default manoeuvre for the selected fault scenario (each fault scenario is associated to a default flight manoeuvre). These runs are distributed evenly among the different fault types applicable to the selected fault scenario (some scenarios contains several sub-scenarios like e.g. different kind of fault profiles). These runs are dedicated to the assessment of the FDD designs performance.

In order to speed up the benchmarking process, the simulations are stopped when a fault is declared as detected by the tested FDD design. The runs with faults are also used for computing the FA rate metric since for these simulations a false alarm can be triggered before the fault is injected, thus the numerator in Eq (2) is $n_{M C}=n_{M C F F+} n_{M C W F}$ while that of Eq (3) is $n_{M C}=n_{M C W F}$.

The Monte Carlo campaign implies random variation within the benchmark defined ranges of the identified geometric parameters (mass, position of the aircraft centre of gravity $\mathrm{Xcg}$ ), the flight parameters (altitude, velocity) and also of the uncertainty in the measurements ( $\Delta$ mass, $\Delta \mathrm{Xcg}, \Delta$ altitude, $\Delta$ velocity) and aerodynamic coefficients $(\Delta \mathrm{CX}, \Delta \mathrm{CY}, \Delta \mathrm{CZ}$, $\Delta \mathrm{Cl}, \Delta \mathrm{Cm}, \Delta \mathrm{Cn})$. This process allows exploring the whole flight domain, a wide class of pilot inputs, perturbations and uncertainties.

\subsection{Validation on Airbus testbeds}

Before validating the more promising FDD designs on the Airbus integration test benches, the corresponding algorithms must be implemented in the FCC.

The state of practice is to write the functional specification, which accurately defines the functions implemented by the FCC software. In a first step, a graphical tool allows specification of these functions through a computer-aided specification. A limited set of graphical symbols (e.g. adder, filter, integrator, look-up tables, etc.) is used to describe each part of the algorithm in dedicated "functional specification sheets". The use of a formal specification language also allows parts of the specification to be used from one aircraft program to another. This specification is under the control of a configuration management tool and its syntax is partially checked automatically. In a second step, an automatic generation tool produces the code to be directly implemented in the FCC [5]. 
For the ADDSAFE project, in order to allow validation of the matured version of the selected FDD designs it is required, in a preliminary step, that they are ported to an AIRBUSspecific Simulink library which uses symbols similar to the graphical language. This allows first to estimate the computational burden of the designs (cf. the ET metric aforementioned) and secondly, it allows to prepare the functional specification sheets. The later are submitted to Airbus-internal V\&V peer review process before automatic coding. The next step is to write the complete laboratory test procedures for ensuring an exhaustive assessment compliant with the required tests.

The results of both, the industrial FES-benchmarking and the Airbus industrial validation are jointly evaluated and a final report, including the comparative results, lessons learnt and conclusions is released to the consortium (whose summary is given in this article).

\section{ASSESSMENT OF DESIGNS}

In this section the summary of the assessment for the developed FDD designs [6-17] is presented. The 13 FDD designs cover techniques such as Sliding-Mode-Observers [6, $11,15], \mathrm{H}$-infinity $[8,9,13]$, linear-parameter-varying [8, 12], Extended Kalman Filters [10], Unknown Input Observers [14] among others.

\subsection{Industrial Verification and Benchmarking}

This activity consisted in applying a Monte Carlo campaign of 2200 runs decomposed into two main cases:

- 1200 fault-free runs distributed evenly (i.e. 200 each) among six benchmark-defined flight maneuvers: cruise, triggering of angle of attack protection, nose-up (abrupt longitudinal maneuver), triggering of pitch protection, coordinated turn and a "yaw-angle-mode" which roughly corresponds to an enhanced auto-pilot hold mode.

- 1000 runs with faults at the default flight manoeuvre for the selected fault scenario. These 1000 runs are distributed evenly among the different types of faults applicable to the fault scenario (e.g. if the aileron fault scenario is being examined [1,2], then 333 runs for liquid jamming, 333 runs for solid jamming and 334 for disconnection).

The first set of cases is used to assess the FA metric (which is the most critical for an actual deployable FDD) while the second looked more specifically to the MD and DTP metrics. In order words, the first case looked at robustness and the second to performance of the FDD designs.

The FES verification procedure is divided in two steps:

- First, the Benchmark \& Verification FES is run, without the FDD designs, for all the required no-fault and fault scenarios in order to obtain the simulation data.

- Second, the appropriate simulation data is injected into each FDD design using "From File" blocks and the results post-processed using the Bench\&Ver-FES.

The simulation data from the first step was made available to all partners subsequently for further maturation and tuning of designs during the $2^{\text {nd }}$ phase of the project (i.e. the Airbus Validation activity).
The FES verification and benchmark results are:

- $\quad$ All the designs but four obtained maximum DTPs well below the desired one.

- All the designs obtained satisfactory $\mathrm{MD}_{\%}$-one case suffered a $0.3 \%$ MD.

- All the designs but one had zero $\mathrm{FA}_{\%}$.

In summary, 9 out of the 13 designs got full marks when using the quantitative DTP, $\mathrm{FA}_{\%}$ and $\mathrm{MD}_{\%}$ metrics and the 4 other designs where close by -suffering only of minor maximum-DTP or $\mathrm{FA}_{\%}$ shortcomings, which has since been corrected. The final selection of the FDD designs for the subsequent industrial validation stage, discussed next, took these quantitative results into account together with the ET metric and the qualitative assessment.

Considering the fastest FCC sampling period (FCC are multirate time triggered digital computers), i.e. $10 \mathrm{~ms}$, the ET estimation results are:

- $\quad$ Three designs obtained ET between 14 and $22 \%$ of the maximum CPU power, which is considered as unrealistic for an implementation in FCC.

- Five designs obtained ET between 3 and 7\% of the maximum CPU capacity which is considered acceptable taking into account that the FCCs used on the most recent aircraft offer more computing capacity.

- $\quad$ Five designs obtained ET between 0.3 and $2 \%$ which is considered as excellent.

The qualitative assessment is more difficult to perform. The number of input parameters to tune oscillates between 6 and more than 40 considering each element of a matrix as a unique input parameter. The physical meaning is also difficult to establish. From an industrial point of view, this is clearly an appealing avenue worth exploring for facilitating the industrial transfer of the proposed FDD designs.

\subsection{Industrial Validation}

When writing the final version of this article, the Airbus industrial validation already started but has not been completed. Initially, the project aimed at assessing the 2 most promising designs. However, the vast majority of the FDD designs submitted by the consortium presented very encouraging results and a low computational load (cf. section 5.1). So, it was decided to test the 5 most promising designs.

The implementation in the FCC will proceed in 3 steps: the first software coding (delivered in May 2012) contains 2 methods, one for abnormal aircraft behaviour scenario (lateral axis) and the other for sensor/actuator malfunctions in the control loop of a control surface (longitudinal axis). A first validation campaign has been done for assessing the robustness of these first 2 designs. In the second FCC coding, expected in June 2012, two additional designs will be implemented for the same scenario, whereas the maturation of the first 2 designs is allowed, taking into account the lesson learnt from the first industrial tests. Finally, the third implementation will add a fifth design dedicated to flight parameter management system. The first 4 designs will benefit from the test results for maturating their robustness and performances. The robustness assessment consists of a series of typical manoeuvres, some of them with a strong 
control surface dynamic: flight control checks, push-over, take-offs in nominal configurations as well as degraded configurations (engine failure, crosswind...), slats/flaps configuration changes, side-step, "duck-under", etc...

The lessons learnt from these first tests are:

- The FCC digital precision is limited and could impact some designs. Especially, the coding of a high-order $(>2)$ filter could be sensitive and lead to error propagation and a diverging behaviour because of coefficient truncation. An adequate filter architecture (e.g. cascade) must be found.

- Since a limited number of symbols can be used inside any functional specification sheet, a relevant and "readable" coding architecture must be found, without creating delays between all sheets dedicated to the same (now split) design.

- A useful and very often used symbol is a look-up table. However, only a limited set of "breaking points" can be used, degrading possibly the design performances.

Finally, a very close collaboration is needed between the designers and the Airbus teams for avoiding any errors that could waste expensive and time-consuming validation on industrial test benches. An upstream, and as complete as possible, design validation is of primary interest.

\section{CONCLUSIONS}

Practical aerospace applications are very challenging due to all the dynamics, perturbations and nonlinearities required to be handled as well as due to all the implementation issues associated with putting on-board an aircraft a certified system. The ADDSAFE project aims to show that the stateof-art in model-based FDD methods is able to tackle the challenges faced in aerospace applications.

From an industrial point of view, two of the strongest points of the ADDSAFE FP7 European project arises due to the representativeness of the Airbus benchmark (consisting of the aircraft model and fault problematic) and the industrial validity of the assessment process. Indeed, the final goal of the project is to assess the developed FDD designs using an industrial FES followed by validation of the more promising designs in the actual Airbus flight control system V\&V setup (from high-fidelity simulation models to the Iron Bird, and including real aircraft actuator rigs). This is a key step for the successful transfer to the aeronautics sector of the FDD methods that have been researched and developed in the academic community in the past decades. This article has detailed the tools, facilities and process followed as well as summarizing the results from its application.

The aerospace community vision for 2050 and beyond promises an aircraft that is still unthinkable, bringing new challenges in term of FDD. This is why such collaboration between the academic world and the industrial practitioners is of primary interest for bridging the gap between basic research levels and technology demonstration.

\section{ACKNOWLEDGMENTS}

This work was performed in the framework of the European ADDSAFE Project: Grant agreement №: FP7-233815.

\section{REFERENCES}

[1] Goupil, P. and Marcos, A. "Advanced Diagnosis for Sustainable Flight Guidance and Control: The European
ADDSAFE Project," SAE AeroTech Congress and Exhibition, October 18-21, doi:10.4271/2011-01-2804.

[2] Goupil, P. and Puyou, G. "A High Fidelity AIRBUS Benchmark for System Fault Detection and Isolation and Flight Control Law Clearance," $4^{\text {th }}$ European Conference for AeroSpace Sciences, July 4-8, 2011.

[3] $11^{\text {th }}$ International Workshop on Simulation \& EGSE facilities for Space Programmes, ESA-ESTEC, 2010, http://cms.dynaweb3.nl/users/esa/?pid=570\&page $=$ Home

[4] Fernandez, V., De Zaiacomo, G., Mafficini, A., Peñín, L. F., "The IXV GNC Functional Engineering Simulator," $11^{\text {th }}$ International Workshop on Simulation \& EGSE facilities for Space Programmes, ESA-ESTEC, 2010

[5] Traverse, P., Lacaze I., Souyris, J., "Airbus fly-by-wire: A total approach to dependability," $18^{\text {th }}$ IFIP World Computer Congress (pp. 191-212), Toulouse, France.

[6] Alwi, H., Edwards, C., "Second Order Sliding Mode Observers for the ADDSAFE Benchmark Problem," SAFEPROCESS 2012

[7] Márton, L., Ossmann D., "Detection of abnormal control surface configurations for civil aircraft," SAFEPROCESS 2012

[8] Henry, D., Cieslak, J., Efimov, D., Zolghadri, A., “A LPV approach for early fault detection in aircraft control surfaces servo-loops," SAFEPROCESS 2012

[9] Vanek, B., Szabó, Z., Edelmayer, A., Bokor, J., "Comparison of geometric and H-infinity fault detection filter design," SAFEPROCESS 2012

[10] Van Eykeren. L., Chu, Q.P., "Sensor fault detection using adaptive extended Kalman filter," SAFEPROCESS 2012

[11] Alwi, H., Edwards, C., "Validation of the sliding mode observer FDI schemes on the ADDSAFE functional engineering simulator," SAFEPROCESS 2012

[12] Varga, A., Ossmann D., "An enhanced LPV-model based identification approach of oscillatory failure cases," SAFEPROCESS 2012

[13] Marcos, A., "Assessment on the ADDSAFE benchmark simulator of H-infinity fault detection designs for aircraft," SAFEPROCESS 2012

[14] Chen, L., Patton, R.J., "Robust fault estimation and performance evaluation based upon the ADDSAFE benchmark model," SAFEPROCESS 2012

[15] Efimov, D., Cieslak, J., Henry, D., Zolghadri, A., "Input estimation via sliding-mode differentiation for early OFC detection," SAFEPROCESS 2012

[16] Vanek, B., Szabó, Z., Edelmayer, A., Bokor, J., "Fault detection of electrical flight control system actuators using geometric methods," SAFEPROCESS 2012

[17] Van Eykeren. L., Chu, Q.P., "Actuator fault detection using aerodynamic model identification," SAFEPROCESS 2012

[18] Goupil, P. "AIRBUS State of the Art and Practices on FDI and FTC in Flight Control System," Control Engineering Practice 19 (2011), pp. 524-539 DOI information: 10.1016/j.conengprac.2010.12.009

[19] Goupil, P. and Marcos, A. "FDD design evaluation matrix”, ADDSAFE report D1.1.2B, Airbus/Deimos, 2011. 